\title{
An Uncertainty Principle for Fermions with Generalized Kinetic Energy
}

\section{Ingrid Daubechies $\star \star \star \star$}

Physics Department, Princeton University, Princeton, NJ 08544, USA

\begin{abstract}
We derive semiclassical upper bounds for the number of bound states and the sum of negative eigenvalues of the one-particle Hamiltonians $h=f(-i \nabla)+V(x)$, acting on $L^{2}\left(\mathbb{R}^{n}\right)$. These bounds are then used to derive a lower bound on the kinetic energy $\sum_{j=1}^{N}\left\langle\psi, f\left(-i \nabla_{j}\right) \psi\right\rangle$ for an $N$-fermion wavefunction $\psi$. We discuss two examples in more detail: $f(p)=|p|$ and $f(p)=\left(p^{2}+m^{2}\right)^{1 / 2}-m$, both in three dimensions.
\end{abstract}

\section{Introduction}

In this paper we present upper bounds for the number of bound states $N(V)$, and the absolute value $S(V)$ of the sum of negative eigenvalues for the single particle Hamiltonians $f(-i \nabla)+V(x)$, acting on $L^{2}\left(\mathbb{R}^{n}\right)$. These bounds are then used to derive a lower bound for the kinetic energy, associated with $f(-i \nabla)$, of a system of $N$ fermions.

In the case where $f(p)=p^{2}$, these bounds are well-known. One has (see e.g. [1], XIII.3 for a review)

$$
\begin{aligned}
N(V) & \leqq C_{n} \int d^{n} x|V(x)|^{n / 2}, & & (n \leqq 3) \\
S(V) & \leqq C_{n}^{\prime} \int d^{n} x|V(x)|^{1+n / 2}, & & (n \leqq 1) .
\end{aligned}
$$

A bound of type (1.2) was first obtained in [2]; later several different and independent proofs for (1.1) were given [3-5]. The best value for the constant $C_{3}$ was obtained in [4].

Using a technique given in $[2,6]$, one can derive from (1.2) the following lower bound on the kinetic energy of an $N$-fermion-system:

\footnotetext{
* Work parially supported by US National Science Foundation Grant PHY-8116101-A01

$\star \star$ On leave from Vrije Universiteit Brussel, and Interuniversitair Instituut voor Kernwetenschappen, Belgium
} 


$$
\sum_{j=1}^{N}\left\langle\psi,\left(-\Delta_{j}\right) \psi\right\rangle \geqq(n / 2)(1+n / 2)^{-1-2 / n}\left(q C_{n}^{\prime}\right)^{-2 / n} \int d^{n} x\left[\rho_{\psi}(x)\right]^{1+2 / n},
$$

where $q$ is the number of possible spin states for the fermions, and where

$$
\rho_{\psi}(x)=N \sum_{\sigma_{i}=1}^{q} \int\left|\psi\left(x, x_{2}, \ldots, x_{N} ; \sigma_{1}, \ldots, \sigma_{N}\right)\right|^{2} d x_{2} \ldots d x_{N},
$$

with $\langle\psi, \psi\rangle=N^{-1} \int d^{n} x \rho_{\psi}(x)=1$.

Bounds of type (1.3) are important for the proof of the stability of matter $[2,6]$. One immediately sees from (1.3) that in order to make the kinetic energy small, the one-particle density $\rho_{\psi}$ has to be "spread out." In this sense (1.3) can be regarded as a mathematical formulation of the physical "uncertainty principle." The derivation of (1.3) uses explicitly the fermion character of the particles (i.e. the asymmetry of $\psi$ ), which is the reason why (1.3) is actually a more constraining inequality than the familiar "uncertainty principle inequality" $\langle\phi,(-\Delta) \phi\rangle \geqq(1 / 4) \int d^{3} x|\phi(x)|^{2}|x|^{-2}$. Unlike (1.3), this last inequality would not be sufficient to establish the stability of matter. (For a detailed discussion, see [6]).

We derive here inequalities analogous to (1.3), but for "generalized" kinetic energy, i.e. for $f(-i \nabla)$, where $f(p)$ need not be $p^{2}$. Our motivation arose again from a stability problem. As an approximation to the study of stability of matter in a relativistic framework, the operator

$$
\begin{aligned}
\sum_{j=1}^{N}\left[\left(-\Delta_{j}+m^{2}\right)^{1 / 2}-m\right] & -\sum_{j=1}^{N} \sum_{k=1}^{K} Z_{k} e^{2}\left|x_{j}-R_{k}\right|^{-1}+\sum_{j<i} e^{2}\left|x_{i}-x_{j}\right|^{-1} \\
& +\sum_{k<l} Z_{k} Z_{l} e^{2}\left|R_{k}-R_{l}\right|^{-1}
\end{aligned}
$$

was studied elsewhere [7]. The problems arising for the case $N=1$ were solved in [7]; for the general problem it seems useful to have a precise bound of the type (1.3) for the kinetic energy operator $\left(-\Delta+m^{2}\right)^{1 / 2}-m$, and the closely related, simpler operator $(-\Delta)^{1 / 2}$ (obtained by setting $m=0$ ). A simple transcription of the $p^{2}$-proof for stability of matter will not work for this case, however, due to the behavior of the lower bound in the kinetic energy in $\int \rho^{4 / 3}$ (see Sect. 3, and [7]). Nevertheless, we hope that the bounds presented here will be useful for solving at least part of the problem.

The paper is organized as follows. In Sect. 2, we use the technique of [4] to derive bounds on $N(V), S(V)$ for kinetic energy operators $f(-i \nabla)$. For our derivation we require that $f(p)$ should be a positive, strictly increasing function of $|p|$ only, with $f(0)=0, \lim _{p \rightarrow \infty} f(p)=\infty$, and such that $f(-i \nabla)$ generates a positivity preserving semigroup. This includes the functions $|p|^{\alpha}(\alpha \leqq 2)$, and $\left(p^{2}+m^{2}\right)^{1 / 2}-m$. The restriction $\alpha \leqq 2$ on $|p|^{\alpha}$ arises from the condition that $f(-i \nabla)$ should generate a positivity preserving semigroup. For the special case $|p|^{\alpha}$ this condition can be dropped, if the desired bounds are derived by the method of [5], which leads however to much larger constants $C_{n}, C_{n}^{\prime}$ (see Remark 3 at the end of Sect. 2).

In Sect. 3 we apply the bounds on $S(V)$ to derive uncertainty inequalities of type 
(1.3) for generalized kinetic energies $f(-i \nabla)$. We calculate explicitly the constants for the case $n=3, f(p)=|p|$ or $\left(p^{2}+m^{2}\right)^{1 / 2}-m$.

\section{Semiclassical Bounds for Hamiltonians with Generalized Kinetic Energy}

We derive in this section semiclassical bounds on the number of negative eigenvalues, and the sum of these eigenvalues, for operators of the form $H=f(-i \nabla)$ $+V(x)$, acting on $L^{2}\left(\mathbb{R}^{n}\right)$.

The semiclassical approximations for these quantities are given by respectively the volume of phase space where $H(p, x)=f(p)+V(x)$ is negative divided by $(2 \pi)^{n}$ (in units where $\hbar=1$, a "cell" of volume $(2 \pi)^{n}$ in $2 n$-dimensional phase space is supposed to correspond to one quantum state), and by the integral of $H$ over this volume.

$$
\begin{aligned}
{[N(V)]_{\mathrm{s}} } & =(2 \pi)^{-n} \int d^{n} x \int d^{n} p \theta[-f(p)-V(x)], \\
{[S(V)]_{\mathrm{s}} } & =\left[\sum\left|e_{j}(V)\right|\right]_{\mathrm{s}}=(2 \pi)^{-n} \int d^{n} x \int d^{n} p \theta[-f(p)-V(x)]|f(p)+V(x)|,
\end{aligned}
$$

where we use the notation $\theta(t)=1$ if $t \geqq 0, \theta(t)=0$ if $t<0$.

We shall assume throughout this section that $V$ is negative: the bounds we shall obtain for $V \leqq 0$ can easily be translated into bounds for general $V$ by replacing $-V$ in our formulae by $\max (0,-V)$. Moreover, we shall always assume $f(p)$ to be a positive, strictly increasing and differentiable function of $|p|$, with inverse function (from $\mathbb{R}_{+}$to $\mathbb{R}_{+}$) denoted by $f^{-1}$. For later use, we also assume $f(0)=0$, and $\lim f(p)=\infty$. $p \rightarrow \infty$

Under these assumptions, the expressions for $[N(V)]_{\mathrm{s} \mathrm{c}}$ and $[S(V)]_{\mathrm{sc}}$ can be rewritten as

$$
\begin{aligned}
& {[N(V)]_{\mathrm{sc}}=k_{n} \int d^{n} x\left[f^{-1}(W(x))\right]^{n},} \\
& {[S(V)]_{\mathrm{sc}}=k_{n} \int d^{n} x \int_{0}^{W(x)} d u\left[f^{-1}(u)\right]^{n},}
\end{aligned}
$$

where $W=-V$, and $k_{n}=\left[n 2^{n-1} \pi^{n / 2} \Gamma(n / 2)\right]^{-1}$.

Our aim here is to show that, provided $f$ satisfies some technical conditions, the real $N(V)$ and $S(V)$ are bounded by these semiclassical approximations, multiplied by a suitable constant (depending on $f$ and on $n$, but not on $V$ ).

Our proof proceeds along the same lines as E. Lieb's proof [4] for the case $f(p)=p^{2}$.

Defining the analogue of the Birman-Schwinger kernel as

$$
K_{\gamma}=W^{1 / 2}[f(-i \nabla)+\gamma]^{-1} W^{1 / 2}, \quad\left(\gamma \in \mathbb{R}_{+}\right),
$$

we have the usual property that

$$
\begin{aligned}
N_{\gamma}(V) & =\text { number of bound states of } f(-i \nabla)+V(x) \text { of energy less than }-\gamma \\
& =\text { number of eigenvalues of } K_{\gamma}>1 .
\end{aligned}
$$

(we assume $V \in C_{0}^{\infty}$ for the time being, which ensures that $V$ is relatively compact with respect to $f(-i \nabla)$, since $\lim _{p \rightarrow \infty} f(p)=\infty$; we shall later consider larger classes for $V$ ). 
Bounds on $N(V), S(V)$ follow from combining the above property of $K_{\gamma}$ with "Lieb's formula" [4]:

$$
\operatorname{Tr}\left[F\left(K_{\gamma}\right)\right]=\int_{0}^{\infty} d t t^{-1} e^{-t} \int d^{n} x \int d \mu_{x, x ; t}(\omega) \phi\left(\int_{0}^{t} d s|V(\omega(s))|\right),
$$

where $\phi$ is a positive, lower semicontinuous function on $[0, \infty)$, where $F$ is defined by $F(x)=\int_{0}^{\infty} d y y^{-1} e^{-y} \phi(x y)$, and $\mu_{x, y ; t}$ is the measure generated by the semigroup $\exp [-t f(-i \nabla)]$, starting at $x$ at time 0 , and pinned at $y$ at time $t$.

E. Lieb's proof of (2.4) (constructed for the case $f(p)=p^{2}$, where the associated measure is a Wiener measure) still works for the general case (the special properties of the Wiener measure were not used), provided $f(-i \nabla)$ generates a positivity preserving semigroup (this is needed in order to construct $\mu_{x, y ; t}$ as a positive measure; the normalization of this measure, i.e. $\int d y \int d \mu_{x, y ; t}=1$, then follows from $f(0)=0$ ).

This will therefore be one of our "technical conditions" on $f$.

Combining (2.3) and (2.4), and using the monotonicity of $F$, we obtain

$$
\begin{aligned}
N_{\gamma}(V) & \leqq[F(1)]^{-1} \operatorname{Tr}\left[F\left(K_{\gamma}\right)\right] \\
& =[F(1)]^{-1} \int_{0}^{\infty} d t t^{-1} e^{-t \gamma} \int d^{n} x \int d \mu_{x, x ; t}(\omega) \phi\left(\int_{0}^{t} d s|V(\omega(s))|\right)
\end{aligned}
$$

Setting $\gamma=0$, this yields a bound on $N(V)$; a bound on $S(V)$ is obtained by using $S(V)$ $=\sum\left|e_{j}\right|=\int_{0}^{\infty} d \gamma N_{\gamma}(V)$.

As in [4], we specialize now to the case where $\phi$ is convex. By Jensen's inequality we have $\phi\left(\int_{0}^{t} W(\omega(s))\right) d s \leqq t^{-1} \int_{0}^{t} \phi(t W(\omega(s))) d s$. After inserting this into (2.5), we can define $\omega(s)=\tilde{\omega}(s)+x$; the measure on the paths $\tilde{\omega}$ is then $\mu_{0,0 ; t}$. For each $s$, we translate the $x$-variable by $\tilde{\omega}(s)$, after which the $s$ - and $\mu$-integrals become trivial, yielding

$$
N_{\gamma}(V) \leqq[F(1)]^{-1} \int_{0}^{\infty} d t t^{-1} e^{-t \gamma} \exp [-t f(-i \nabla)](0,0) \int d^{n} x \phi(t W(x)) .
$$

Using $\exp [-t f(-i \nabla)](0,0)=(2 \pi)^{-n} \int d^{n} p e^{-t f(p)}$, this finally leads to

$$
\begin{gathered}
N(V) \leqq k_{n}^{\prime} \int d^{n} x g_{1}(W(x), \phi), \\
S(V) \leqq k_{n}^{\prime} \int d^{n} x g_{2}(W(x), \phi),
\end{gathered}
$$

where

and

$$
k_{n}^{\prime}=\left[2^{-1+n / 2} \Gamma(n / 2)(2 \pi)^{n / 2}\right]^{-1}=n k_{n}
$$

$$
g_{j}(W, \phi)=[F(1)]^{-1} \int_{0}^{\infty} d t t^{-j} \int_{0}^{\infty} d p p^{n-1} e^{-t f(p)} \phi(t W) .
$$

The inequalities (2.6) have to be compared with (2.1), (2.2). 
We shall restrict ourselves from now on to a one-parameter family of $\phi-s$ :

$$
\phi_{a}(u)=\left\{\begin{array}{ll}
0 & u \leqq a \\
u-a & u \geqq a
\end{array} \quad(a>0)\right.
$$

with associated $F_{a}=F\left(\phi_{a} ; 1\right)=a e^{-a} \int_{0}^{\infty} d y y(y+1)^{-1} e^{-a y}$.

We denote the associated $g_{j}\left(W, \phi_{a}\right)$ by $g_{j}(W, a)$, and we show below that

$$
g_{1}(W, a) \leqq C_{1}(a)\left[f^{-1}(W)\right]^{n}
$$

and

$$
g_{2}(W, a) \leqq C_{2}(a) \int_{0}^{W} d \beta\left[f^{-1}(\beta)\right]^{n},
$$

which then implies that $N(V), S(V)$ are bounded by $K_{1}[N(V)]_{\mathrm{sc} \text {. }}$, $K_{2}[S(V)]_{\text {s.c. }}$ respectively.

It is obvious that $g_{1}(0, a)=g_{2}(0, a)=0$. Since $\phi_{a}^{\prime}(u)=0$ for $u<a$, and $\phi_{a}^{\prime}(u)=1$ for $u>a$, we have (it can be checked that the $g_{j}$ are differentiable provided the integrals below converge)

$$
\begin{aligned}
\frac{d}{d W} g_{j}(W, a) & =\left(1 / F_{a}\right) \int_{a / W}^{\infty} d t t^{-j+1} \int_{0}^{\infty} d p p^{n-1} e^{-t f(p)} \\
& =\left(1 / F_{a}\right) \int_{0}^{\infty} d p p^{n-1}[f(p)]^{j-2} \int_{a f(p) / W}^{\infty} d t t^{-j+1} e^{-t} .
\end{aligned}
$$

For $j=1$ this leads to

$$
\frac{d}{d W} g_{1}(W, a)=\left(1 / F_{a}\right) \int_{0}^{\infty} d p\left[p^{n-1} / f(p)\right] e^{-a f(p) / W}
$$

or

$$
g_{1}(W, a)=\left(1 / F_{a}\right) \int_{0}^{\infty} d p\left[p^{n-1} / f(p)\right] \int_{0}^{W} d \beta e^{-a f(p) / \beta} .
$$

Substituting $u=a f(p) / \beta$ and inverting the order of the integrals, this reduces to

$$
g_{1}(W, a)=\left(a / n F_{a}\right) \int_{0}^{\infty} d u e^{-u} u^{-2}\left[f^{-1}(W u / a)\right]^{n}
$$

For $j=2$, (2.9) leads directly to

$$
\frac{d}{d W} g_{2}(W, a)=\left(n F_{a}\right)^{-1} \int_{0}^{\infty} d u e^{-u} u^{-1}\left[f^{-1}(W u / a)\right]^{n} .
$$

Define $\quad \psi_{f}(y)=\sup \left[f^{-1}(x y) / f^{-1}(x)\right]$. Replacing $f^{-1}(W u / a)$ by $f^{-1}(W) \psi_{f}(u / a)$ obviously leads to upper bounds of the type (2.8) for the $g_{j}$, which then in turn imply

$$
N(V) \leqq K_{1}[N(V)]_{\mathrm{s} \mathrm{c}}, \quad S(V) \leqq K_{2}[S(V)]_{\mathrm{s} \mathrm{c}},
$$


with

$$
K_{j}=\inf _{a>0}\left(a^{-1} e^{a}\left\{\int_{0}^{\infty} d u e^{-a u} u^{-3+j}\left[\psi_{f}(u)\right]^{n}\right\}\left\{\int_{0}^{\infty} d u e^{-a u} u(u+1)^{-1}\right\}^{-1}\right)
$$

Inequality (2.10) bounds $N(V), S(V)$ by their semiclassical approximations, which was our aim. We have taken $V$ in $C_{0}^{\infty}$, and assumed the following properties for $f: f(p)$ is a differentiable, positive function only depending on $|p|$, strictly increasing in $|p|$, with $f(0)=0, \lim _{p \rightarrow \infty} f(p)=\infty ;$ moreover $f(-i \nabla)$ generates a positivity preserving semigroup, and $\psi_{f}(y)=$ $\sup \left[f^{-1}(x y) / f^{-1}(x)\right]$ should be such that the integral in (2.11) converges for at least $x>0$ some $a$.

\section{Remarks}

1. Of the conditions on $f$, the restriction that $f$ has to generate a positivity preserving semigroup seems the least easy to verify. Actually, there exist several criteria to verify this property: see e.g. [1], p. 209-222. Also, once a positive function $f$ is given which generates a positivity preserving semigroup, the composition $F \circ f$ of any positive $C^{\infty}$-function $F$ on $\mathbb{R}_{+}$, satisfying $(-1)^{n+1} F^{(n)}(x) \geqq 0, n=1,2, \ldots$, with $f$, will again generate a positivity preserving semigroup (see [8]; one can check this easily by verifying that the condition on $F$ implies that $e^{-F}$ is completely monotone, and then applying Bernstein's theorem to $\left.e^{-F}\right)$. In particular, since $e^{t \Delta}$ is positively preserving for all $n$, the functions $f_{\alpha}(p)=|p|^{\alpha}, \alpha \in[0,2]$ and $k(p)=\left[p^{2}+m^{2}\right]^{1 / 2}-m$ generate positivity preserving semigroups, for all $n$.

2. For these functions $f_{\alpha}, k$, one has $\psi_{f_{\alpha}}(y)=y^{1 / \alpha}, \psi_{k}(y)=\max \left(y^{1 / 2}, y\right)$ (for $f_{\alpha}$, this is trivial; for $k$ it follows from an easy calculation). Hence $K_{2}$ is finite for both $f_{\alpha}$ and $k$ in all dimensions $n ; K_{1}$ is finite for $k$ only if $n \geqq 3$, and for $f_{\alpha}$ if $n>\alpha$, i.e. for all $\alpha$ if $n \geqq 3$, for $\alpha<2$ if $n=2$, and for $\alpha<1$ if $n=1$. Since all the other conditions hold for $f_{\alpha}$ and $k$, the bounds (2.10) hold for these functions.

3. The restriction that $f_{\alpha}$ has to generate a positivity preserving semigroup prevents us from going beyond $\alpha=2$ for the $f_{\alpha}$-functions. Actually this restriction only arises because our method of proving (2.10) uses a path integral; with other methods one can show that a nontrivial (i.e. with finite constant $K_{1}$ ) bound of type (2.10) holds for $N(V)$ for $f_{\alpha}(p)$ as long as $\alpha<n$, without any other restriction on $\alpha$. (The fact that for $\alpha=n$ such a bound does not exist is the generalization of the familiar nonexistence of this bound for $p^{2}+V(x)$ in 2 dimensions. In $n$ dimensions also, the Hamiltonian $p^{n}+V(x)$, where $V$ is a square well, always has bound states, however shallow the well.) One such proof is provided by Cwikel's estimate [5]: any $A=f(x) g(-i \nabla)$, with $f \in L^{p}, g \in L_{w}^{p}$ with $2<p<\infty$, is a bounded operator with singular values $\mu_{k}(A)$ obeying

$$
\mu_{k}(A) \leqq D_{p, n}\|f\|_{p}\|g\|_{p, w}^{*} k^{-1 / p}
$$


where $D_{p, n}=2+\left((2 \pi)^{-n} 2^{p}\left[1-2^{-(p-2)}\right]^{-1}\right)^{1 / 2}$. This allows one to give an upper bound on the number of eigenvalues of $|V|^{1 / 2}|p|^{-\alpha}|V|^{1 / 2}$ which are larger than 1 , hence a bound on $N(V)$ :

$$
N(V) \leqq\left[D_{2 n / \alpha, n}\right]^{2 n / \alpha}(2 \pi)^{n}[N(V)]_{\mathrm{s} \mathrm{c}}
$$

For $\alpha \leqq 2$ we can compare this constant with (2.11) for the special case $f=f_{\alpha}$ :

$$
K_{1}(\alpha, n)=\Gamma(-1+n / \alpha) \inf _{a>0}\left[a^{1-n / \alpha}\left(\int_{a}^{\infty} d u e^{-u}(u-a) u^{-1}\right)^{-1}\right] .
$$

For $n=3, \alpha=2, K_{1}(\alpha)$ is a much better constant than the one in (2.13) (E. Lieb's proof was constructed to treat this case; the bound he obtained is still the best bound at present); from numerical calculations for some other values of $(n, \alpha)$ it seems that this will be generally true for $\alpha \leqq 2$.

It turns out that a suitable modification of Cwikel's proof for (2.12) can also handle the case $k(p)=\left(p^{2}+m^{2}\right)^{1 / 2}-m$. The bound for $N(V)$ obtained in this way is of the following type: $N(V) \leqq C \int d^{n} x G(|V(x)|)$, where $G(\alpha) \underset{\alpha \rightarrow \infty}{\sim} \alpha^{n}$, $G(\alpha) \underset{\alpha \rightarrow 0}{\sim} C^{\prime} \alpha^{n / 2}$. This is also what we obtain by our method. Again however, the constant $C$ obtained in this way seems to be at least two orders of magnitude larger than with the path integral method.

4. The method leading to the bounds (2.10) on $N(V), S(V)$ can also be used to derive bounds on other momenta of the eigenvalues, i.e. $\sum\left|e_{j}\right|^{s}$, with $s \neq 0$ or 1. Explicitly, one obtains

$$
\sum\left|e_{j}\right|^{s} \leqq C_{s} \int d^{n} x G_{s}(W(x)),
$$

with

$$
\begin{aligned}
G_{s}(W)= & \int_{0}^{W} d \beta\left[f^{-1}(\beta)\right]^{n} \beta^{s-1}, \quad(s>0), \\
C_{s}= & \Gamma(s)\left[2^{n-1} \pi^{n / 2} \Gamma(n / 2)\right]^{-1} \inf _{a>0}\left[\left(\int_{a}^{\infty} d t(t-a) t^{-1} e^{-t}\right)^{-1}\right. \\
& \left.\left(\int_{0}^{\infty} d t t^{-s} e^{-t} \int_{0}^{\psi_{f}^{(t / a)}} d u u^{n-1} \phi_{f}(u)^{s-1}\right)\right],
\end{aligned}
$$

where we have introduced another auxiliary function: $\phi_{f}(y)=\sup _{x>0}[f(x y) / f(x)]$. In the case $f(p)=|p|^{\alpha},(2.14)$ reduces to bounds of the type $\sum\left|e_{j}\right|^{s} \leqq$ $C_{s, \alpha}^{\prime} \int d^{n} x|W(x)|^{s+n / \alpha}$.

5. We have restricted ourselves, in the derivation of the bounds (2.10), to $C_{0}^{\infty}$ potentials. Of course (2.10) will generally be valid for larger classes of potentials, and ideally, we would like it to be true for all potentials for which the semiclassical approximations (2.1) and (2.2) converge. For $f(p)=|p|^{\alpha}$, one can indeed show this is the case. Inequalities (2.10) or (2.13) can then be written as

$$
\text { for } V \in C_{0}^{\infty}, \quad V \leqq 0: N(V) \leqq K_{\alpha}^{\prime} \int d^{n} x|V(x)|^{n / \alpha} .
$$

For any nonpositive $V$ in $L^{n / \alpha}$, one can then find $V_{m}$ in $C_{0}^{\infty}$ such that $\left\|V_{m}-V\right\|_{n / \alpha} \rightarrow 0$ 
and $\left|V_{m}\right| \leqq|V|$ a.e.. One can then check that $|p|^{\alpha}+V_{m} \rightarrow|p|^{\alpha}+V$ in the norm resolvent sense, which implies traceclass convergence of the spectral projections of these operators on $(\infty, E]$ for any $E<0$, which in turn then implies (2.15) for $V$ (with the same constant $K_{\alpha}^{\prime}$ ). An analogous argument shows that for $k(p)=\left(p^{2}+m^{2}\right)^{1 / 2}-m$, the bound $N(V) \leqq K \int d^{n} x[|V(x)|(|V(x)|+2 m)]^{n / 2}$ is valid for all nonpositive $V$ in $L^{n / 2} \cap L^{n}$. Analogously one proves that the bound on $S(V)$ in (2.10) holds for all $V \in L^{1+n / \alpha}$ if $f(p)=|p|^{\alpha}$, for all $V \in L^{1+n / 2} \cap L^{n+1}$ if $f(p)=\left(p^{2}+m^{2}\right)^{1 / 2}-m$.

6. As we pointed out in the introduction, the cases of most interest to us are $n=3, f(p)=|p|$ or $f(p)=\left(p^{2}+m^{2}\right)^{1 / 2}-m$. We have therefore calculated the constants $K_{1}, K_{2}$ numerically for these two cases. The results are:

$$
\begin{aligned}
\text { for } f(p)=|p|, \quad n=3: & K_{1} \leqq 6.08 ; \quad k_{3} K_{1} \leqq 0.103 \\
& K_{2} \leqq 6.44 ; \quad k_{3} K_{2} \leqq .109 \\
\text { for } f(p)=\left(p^{2}+m^{2}\right)^{1 / 2}-m, \quad n=3: \quad & K_{1} \leqq 14.14 ; \quad k_{3} K_{1} \leqq 0.239 \\
& K_{2} \leqq 9.62 ; \quad k_{3} K_{2} \leqq .163
\end{aligned}
$$

(where $\left.k_{3}=\left(6 \pi^{2}\right)^{-1}\right)$. Note that one can easily prove that $\sup ([S(V)] /$ $\left.[S(V)]_{\text {s.c. }}\right)$ is always smaller than $\sup \left([N(V)] /[N(V)]_{\text {s.c }}\right.$ ) (any bound on $N(V)$ gives a bound on $S(V)$ by simple integration). This shows that the upper bound on $K_{2}$ for $f(p)=|p|$ above is not very good, since it is larger than the corresponding $K_{1}$; in practical applications, we shall therefore use the above value for $K_{1}$ for bounds on $S(V)$.

\section{Bounds on the Kinetic Energy for Fermions}

Once an upper bound of type (2.10) is known for $S(V)$, the sum of the negative eigenvalues of $f(-i \nabla)+V(x)$, it is easy to derive from this a lower bound on the fermionic kinetic energy associated with $f(-i \nabla)$. This is done by means of a technique which can be found in [2].

Let $\psi\left(x_{1}, \ldots, x_{N} ; \sigma_{1}, \ldots, \sigma_{N}\right)$ be a normalized wave function for $N$ fermions. Consider, for suitable negative functions $V$ on $\mathbb{R}^{n}(V$ is "suitable" essentially if $[S(V)]_{\text {s.c. }}$ is finite), the $N$-particle Hamiltonian $H_{N}=\sum_{j=1}^{N} h_{j}$, with $h_{j}=f\left(-i \nabla_{j}\right)+V\left(x_{j}\right)$. Assuming the fermions have $q$ possible spin states, we have that the ground state $E_{0}$ of $H_{N}$ satisfies $E_{0} \geqq q \sum_{k} e_{k}(h)$, where $e_{k}(h)$ are the negative eigenvalues of the single particle Hamiltonian $h$. If $h$ has $K$ levels, and $N \leqq K q$, we surely have $E_{0} \geqq q \sum e_{k}$; if $N>K q$, we fill the available $K q$ levels, and place the excess particles far away, with arbitrary small energy, which again ensures $E_{0} \geqq q \sum e_{k}$.

Hence, using (2.10) and (2.2),

$$
\left\langle\psi, \sum_{j} f\left(-i \nabla_{j}\right) \psi\right\rangle-\int d^{n} x|V(x)| \rho(x) \geqq E_{0} \geqq-q K_{2} k_{n} \int d^{n} x F(|V(x)|),
$$


where

$$
\rho(x)=N \sum_{\sigma_{i}=1}^{q} \int\left|\psi\left(x, x_{2}, \ldots, x_{N} ; \sigma_{1}, \ldots, \sigma_{N}\right)\right|^{2} d x_{2} \ldots d x_{N}
$$

and $F(s)=\int_{0}^{s} d t\left[f^{-1}(t)\right]^{n}$. This implies that a lower bound on the kinetic energy $T_{\psi}$ is given by

$$
T_{\psi}=\left\langle\psi, \sum_{j} f\left(-i \nabla_{j}\right) \psi\right\rangle \geqq \int d^{n} x \mathscr{L}(C F)(\rho(x)),
$$

where $C=q K_{2} k_{n}$, and where $\mathscr{L}$ denotes the Legendre transform

$$
\mathscr{L}(C F)(t)=\sup _{s}\left\{s t-C \int_{0}^{s} d u\left[f^{-1}(u)\right]^{n}\right\} .
$$

The maximum in $s$ is reached for $s=f\left[(t / C)^{1 / n}\right]$. Using partial integration, this leads to

$$
\mathscr{L}(C F)(t)=C \int_{0}^{t / C} d u f\left(u^{1 / n}\right)
$$

Hence (3.1) becomes

$$
T_{\psi}=\left\langle\psi, \sum_{j} f\left(-i \nabla_{j}\right) \psi\right\rangle \geqq C \int d^{n} x \int_{0}^{\rho(x) / C} d u f\left(u^{1 / n}\right) .
$$

For the special case $f(p)=|p|^{\alpha},(3.2)$ can be written more explicitly. We have

$$
\left\langle\psi, \sum_{j=1}^{N}\left|p_{j}\right|^{\alpha} \psi\right\rangle \geqq C_{\alpha, n} \int d^{n} x[\rho(x)]^{1+\alpha / n},
$$

where

$$
C_{\alpha, n}=[1+\alpha / n]^{-1}\left[q k_{n} K_{2}(n, \alpha)\right]^{-\alpha / n}
$$

with $k_{n}=\left[n 2^{n-1} \pi^{n / 2} \Gamma(n / 2)\right]^{-1}$ and

$$
K_{2}(n, \alpha)=\Gamma(n / \alpha) \inf _{a>0}\left[a^{-n / \alpha}\left(\int_{a}^{\infty} d u e^{-u}(u-a) u^{-1}\right)^{-1}\right] .
$$

For the special case $n=3, \alpha=1,(3.3)$ becomes

$$
\left\langle\psi, \sum_{j=1}^{N}\left|p_{j}\right| \psi\right\rangle \geqq 1.63 q^{-1 / 3} \int d^{3} x \rho(x)^{4 / 3} .
$$

We can also work out (3.2) for the case $n=3, f(p)=\left(p^{2}+m^{2}\right)^{1 / 2}-m$. It turns out that

$$
\left\langle\psi, \sum_{j=1}^{N}\left[\left(p_{j}^{2}+m^{2}\right)^{1 / 2}-m\right] \psi\right\rangle \geqq \int d^{3} x G[\rho(x)],
$$

where $G(\rho)=(3 / 8) m^{4} C g\left[(\rho / C)^{1 / 3} m^{-1}\right]-m \rho$ with

$$
g(t)=t\left(1+t^{2}\right)^{1 / 2}\left(1+2 t^{2}\right)-\ln \left[t+\left(1+t^{2}\right)^{1 / 2}\right]
$$


and $C=.163 q$. It can be checked that $G$ is convex (as it should be); the asymptotic behavior of $G$ for small, respectively large $\rho$ is given by

$$
G(\rho) \underset{\rho \rightarrow 0}{\sim}(3 / 10 m) C^{-2 / 3} \rho^{5 / 3}, G(\rho) \underset{\rho \rightarrow \infty}{\sim}(3 / 4) C^{-1 / 3} \rho^{4 / 3} .
$$

The $\rho^{4 / 3}$ behavior of $G$ for large $\rho$ (i.e. small $x$ ) reflects the fact that for large $|p|,\left[p^{2}+m^{2}\right]^{1 / 2}-m$ behaves like $|p|$ (we saw in (3.4) that the bound for $T_{\psi}$ is in $\rho^{4 / 3}$ for $\left.f(p)=|p|\right)$. On the other hand $G$ behaves like $m^{-1} \rho^{5 / 3}$ if $\rho$ is small (i.e. for large $x$ ), which is due to the fact that for small $|p|$, $\left[p^{2}+m^{2}\right]^{1 / 2}-m \sim p^{2} / 2 m$.

Acknowledgements. I wish to thank here E. Lieb for suggesting that the methods of [4] might be used for the relativistic kinetic energy operator $\left[p^{2}+m^{2}\right]^{1 / 2}-m$, for stimulating my interest in these problems, and for many interesting and helpful discussions. I also want to thank M. Loss for several helpful discussions, and B. Simon for showing me that Cwikel's method can be used to derive a bound on $N(V)$ for the kinetic energy operator $k(p)=\left(p^{2}+m^{2}\right)^{1 / 2}-m$.

\section{References}

1. Reed, M., Simon, B.: Methods of modern mathematical physics, Vol. IV: Analysis of operators. New York: Academic Press 1978

2. Lieb, E., Thirring, W.: A bound for the kinetic energy of fermions which proves the stability of matter. Phys. Rev Lett. 35, 687-689 (1975). More details are given in Lieb, E., Thirring, W.: Inequalities for the moments of the eigenvalues of the Schrödinger Hamiltonian and their relation to Sobolev inequalities. In: Studies in Mathematical Physics: Essays in Honor of Valentine Bargmann, Lieb, E. H., Simon, B., Wightman, A. S., (eds.). Princeton: Princeton University Press 1976

3. Rosenbljum, G.: The distribution of the discrete spectrum for singular differential operators. Dokl. Akad. Nauk SSSR 202 (1972) (Transl. Sov. Math. Dokl. 13, 242-249 (1972))

4. Lieb, E.: Bounds on the eigenvalues of the Laplace and Schrödinger operators. Bull. Am. Math. Soc. 82, 751-753 (1976). More details can be found in Lieb, E.: The number of bound states of one-body Schrödinger operators and the Weyl problem. Proc. Am. Math. Soc. 36, 241-252 (1980)

5. Cwikel, M.: Weak type estimates and the number of bound states of Schrödinger operators. Ann. Math. 106, 93-102 (1977)

6. Lieb, E.: The stability of matter. Rev. Mod. Phys. 48, 553-569 (1976)

7. Daubechies, I., Lieb, E.: One-electron relativistic molecules with Coulomb interaction. Commun. Math. Phys. 90, (1983)

8. Bratteli, O., Kishimoto, A., Robinson, D.: Positivity and monotonicity of $C_{0}$-semigroups, I. Commun. Math. Phys. 75, 67-84 (1980)

Communicated by B. Simon

Received April 18, 1983 